\title{
The (ir)Relevance of Initial Conditions in Soft Leptogenesis
}

\author{
Omri Bahat-Treidel and Ze'ev Surujon \\ Department of Physics, Technion - Israel Institute of Technology, \\ Haifa 32000, Israel
}

\begin{abstract}
We explore how the initial conditions affect the final lepton asymmetry in Soft Leptogenesis. It has been usually assumed that the initial state is a statistical mixture of sterile sneutrinos and anti sneutrinos with equal abundances. We calculate the lepton asymmetry due to the most general initial mixture. The usually assumed equal mixture produces a small, but sufficient, lepton asymmetry which is proportional to the ratio of the supersymmetry breaking scale over the Majorana scale. A more generic mixture, still with equal contents of sneutrinos and anti sneutrinos, yields an unsuppressed lepton asymmetry. Mixtures of non equal contents of sneutrinos and anti sneutrinos result in a large lepton asymmetry too. While these results establish the robustness of Soft Leptogenesis and other mixing based mechanisms, they also expose their lack of predictive power.
\end{abstract}




\section{INTRODUCTION}

The accepted paradigm in baryogenesis relies on generating baryon asymmetry dynamically from symmetric initial conditions. Such generation may occur if there are processes which satisfy the Sakharov conditions [1], namely, they violate baryon number, they violate $\mathrm{C}$ and $\mathrm{CP}$, and they occur out of equilibrium. In Leptogenesis scenarios [2], a lepton asymmetry is generated at a high temperature, and is partially transformed into a baryon asymmetry by the $(B-L)$-violating sphalerons. The $L, \mathrm{C}$ and $\mathrm{CP}$ violation may come from various sources, such as the decays of sterile neutrinos [2, 3] or sneutrinos [4, 5], the mixing between them (Resonant Leptogenesis [6] and Soft Leptogenesis [8, 9, 10]), or interference effects [11]. The third condition is satisfied by the decaying of the heavy particles (sterile neutrinos and sneutrinos), which occurs out of equilibrium once the temperature becomes low enough.

For simplicity, we limit ourselves to Soft Leptogenesis, which is based on the minimal supersymmetric standard model plus a sterile neutrino superfield $N$. In this framework, the soft supersymmetry breaking parameters are responsible for the CP violation ${ }^{1}$. The leading contribution [8, 10] comes from $\mathrm{CP}$ violation in the mixing between the sneutrino $(\tilde{N})$ and the anti-sneutrino $\left(\tilde{N}^{\dagger}\right)$, and therefore it is present already in a model with one generation of leptons.

The lepton asymmetry produced in this mechanism depends on the state of the $\tilde{N}-\tilde{N}^{\dagger}$ system before decaying. It has been usually assumed in the literature that this initial state is an equal incoherent mixture of $|\tilde{N}\rangle$ and $\left|\tilde{N}^{\dagger}\right\rangle$. Although this seems to be a reasonable assumption, the actual initial state could be different, since it depends on the couplings of the sneutrinos in the high energy theory, which determine both the details of the sneutrino production and the physics responsible for the decoherence of the plasma. For example, the sneutrinos may be produced non-thermally by direct inflaton decay [4, 13 . In this case, the coupling to the inflaton is needed in order to determine the plasma state just before Leptogenesis occurs; In Grand Unified Theories, the initial state would depend on the way the neutrino superfield is embedded in the matter multiplet; In models which explain the flavor hierarchy, the coupling to the new fields (e.g. flavons) and the sneutrino representation under the flavor group could largely affect the initial state, and so on. Previous discussions of the initial state can be found for example in [4, 7, 13, 14].

In this work, we give a general treatment of this issue, by considering a generic initial state. We find that the resulting lepton asymmetry is generally enhanced compared to the

1 We should mention here that the idea of using supersymmetry breaking terms for Leptogenesis was raised already in an earlier work [12] in the context of low scale Leptogenesis. 
one resulting from the usually assumed initial state. As particular cases, we consider a mixture of "CP eigenstates" $|\tilde{N}\rangle \pm e^{i \phi}\left|\tilde{N}^{\dagger}\right\rangle$. As in meson mixing, this labeling is appropriate in the presence of small CP violation. These mixtures have, as in the case of the usually assumed state, equal expectation values of $\tilde{N}$ and $\tilde{N}^{\dagger}$. Such mixtures yield an enhanced asymmetry too. A motivation for considering such mixtures would be an approximate CP conservation of the high energy theory.

Another particular case is a mixture of the Hamiltonian eigenstates which, if the CP violation is small, are very similar to the Hamiltonian eigenstates mentioned above. Such mixtures would be motivated by assuming that the quantum decoherence is due to the same Hamiltonian, so that only the energy eigenstates are projected in. This mixture also yields an enhanced lepton asymmetry.

Since our calculations rely on $\mathrm{CP}$ violation in mixing, we expect them to be valid in any mixing based Leptogenesis, e.g. Resonant Leptogenesis [6].

\section{DEFINITIONS AND APPROXIMATIONS}

As stated above, in this work we consider the Soft Leptogenesis scenario [8, 9], which employs the minimal supersymmetric standard model plus singlet neutrino chiral superfields. Since Soft Leptogenesis requires only one generation, the relevant part of the superpotential is

$$
W=\frac{1}{2} M N N+Y L N H_{u},
$$

where we use the notation of [8]. Soft supersymmetry breaking is introduced by

$$
\mathcal{L}_{\text {soft }}=B \tilde{N} \tilde{N}+A \tilde{L} \tilde{N} H_{u}
$$

where $\tilde{N}$ is the singlet sneutrino. The soft breaking parameters are assumed, as usual, to be highly suppressed:

$$
\frac{B}{M^{2}} \ll 1, \quad \frac{A}{M} \ll 1 .
$$

Yet, they may have a significant effect proportional to the $\mathrm{CP}$ violating phase $\varphi \equiv \arg \left(A M B^{*} Y^{*}\right)$. This weak phase shows up in decays of $\tilde{N}$ and $\tilde{N}^{\dagger}$, in the mixing between them and in the interference between decays with and without oscillations. In most of the parameter space, though, the largest effect is due to CP violation in mixing.

In analogy to meson mixing and decay, we define

$$
x \equiv \frac{\Delta m}{\Gamma}, \quad y \equiv \frac{\Delta \Gamma}{2 \Gamma},
$$


where $\Delta m$ and $\Delta \Gamma$ are the mass and width differences of the mass eigenstates

$$
\left|\tilde{N}_{L, H}\right\rangle=p|\tilde{N}\rangle \pm q\left|\tilde{N}^{\dagger}\right\rangle, \quad \frac{q}{p} \equiv R e^{i \phi}
$$

Note that $\phi$ depends on convention. In particular, in the convention where the decay amplitudes are real, we have $\phi=-\varphi$. We also define

$$
\epsilon \equiv 1-R^{2}
$$

which signals $\mathrm{CP}$ violation in mixing. Note that $\epsilon$ is expected to be small,

$$
\epsilon=\mathcal{O}\left(\epsilon_{S} / x\right), \quad \epsilon_{S} \equiv \frac{m_{\mathrm{SUSY}}}{M}
$$

(see [11], note slight difference in notations).

We denote the relevant amplitudes by

$$
\begin{aligned}
A_{\tilde{L}} \equiv A(\tilde{N} \rightarrow \tilde{L} H), & A_{\bar{L}} \equiv A(\tilde{N} \rightarrow \bar{L} \bar{h}), \\
\bar{A}_{\tilde{L}^{\dagger}} \equiv A\left(\tilde{N}^{\dagger} \rightarrow \tilde{L}^{\dagger} H^{\dagger}\right), & \bar{A}_{L} \equiv A\left(\tilde{N}^{\dagger} \rightarrow L h\right) .
\end{aligned}
$$

Following [8], we make the following approximations (these approximations are relaxed in [11] and in the appendix of this article):

1. $A_{\tilde{L}}=\bar{A}_{\tilde{L}^{\dagger}}$.

2. $A_{\bar{L}}=\bar{A}_{L}$.

3. All the two body decay amplitudes are negligible except those in Eq. (8)).

4. We neglect three body decays (this is justified in [11]).

The lepton asymmetry due to the usually assumed initial state (a mixture of $|\tilde{N}\rangle$ and $\left|\tilde{N}^{\dagger}\right\rangle$ with equal abundances) is found in [8, 9] to be

$$
\varepsilon=\frac{\tilde{\mathcal{N}}\left|A_{\tilde{L}}\right|^{2}-\mathcal{N}\left|A_{\bar{L}}\right|^{2}}{\tilde{\mathcal{N}}\left|A_{\tilde{L}}\right|^{2}+\mathcal{N}\left|A_{\bar{L}}\right|^{2}}\left(\frac{x^{2}+y^{2}}{1+x^{2}}\right) \epsilon
$$

where $\tilde{\mathcal{N}}$ and $\mathcal{N}$ are phase space factors (see [8]). In this work, we show how this result emerges from the more general expression as a special case.

A further generalization would be to consider other types of $\mathrm{CP}$ violation [11]. This requires considering gaugino mass insertions and relaxing the assumptions concerning amplitudes. In the appendix we show some consequences of this generalization. 


\section{THE INITIAL STATE}

Our Hilbert space is two dimensional, and thus an arbitrary mixture of $k$ pure states can always be described by a $2 \times 2$ density matrix. Therefore, it is physically equivalent to some (non-equal) mixture of two orthogonal states

$$
\begin{aligned}
& |\psi\rangle=e^{i \beta}\left(\cos \theta|\tilde{N}\rangle-e^{i \alpha} \sin \theta\left|\tilde{N}^{\dagger}\right\rangle\right), \\
& |\bar{\psi}\rangle=e^{i \gamma}\left(e^{-i \alpha} \sin \theta|\tilde{N}\rangle+\cos \theta\left|\tilde{N}^{\dagger}\right\rangle\right)
\end{aligned}
$$

which are the eigenstates of the density matrix. We further define $n$ and $\bar{n}$ to be the statistical abundances of $|\psi\rangle$ and $|\bar{\psi}\rangle$, respectively.

The overall phase $(\beta+\gamma)$ is not observable in quantum mechanics. Furthermore, considering statistical mixtures of $|\psi\rangle$ and $|\bar{\psi}\rangle$ means that we cannot measure the relative phase $(\beta-\gamma)$ as well. Therefore, a statistical mixture is described by three free parameters, which are $\theta, \alpha$, and $n$ (the statistical abundance of $|\psi\rangle$ ). Indeed, being Hermitian and traceless, a two dimensional density matrix has three real parameters. In our parameterization, the density matrix takes the form

$$
\begin{aligned}
\rho=n|\psi\rangle\langle\psi|+\bar{n}| \bar{\psi}\rangle\langle\bar{\psi}| & =\left(\begin{array}{cc}
n \cos ^{2} \theta+\bar{n} \sin ^{2} \theta & -(n-\bar{n}) e^{-i \alpha} \cos \theta \sin \theta \\
-(n-\bar{n}) e^{i \alpha} \cos \theta \sin \theta & n \sin ^{2} \theta+\bar{n} \cos ^{2} \theta
\end{array}\right) \\
& =\frac{1}{2}\left(\begin{array}{cc}
1+c \Delta & -s e^{-i \alpha} \Delta \\
-s e^{i \alpha} \Delta & 1-c \Delta
\end{array}\right),
\end{aligned}
$$

where

$$
s \equiv \sin (2 \theta), \quad c \equiv \cos (2 \theta), \quad \Delta \equiv n-\bar{n} .
$$

We denote by $|\tilde{N}\rangle$ and $\left|\tilde{N}^{\dagger}\right\rangle$ the sneutrino "flavor" states. Then, the diagonal elements are just the expectation values of the "flavor" state abundances $\left(\left\langle n_{f}\right\rangle,\left\langle\bar{n}_{f}\right\rangle\right)$. We also define

$$
\Delta_{f} \equiv\left\langle n_{f}\right\rangle-\left\langle\bar{n}_{f}\right\rangle=\cos (2 \theta) \Delta
$$

as a measure of "flavor" imbalance. A particularly interesting type of initial states is one which concerns "flavorless" mixtures, i.e. mixtures with $\Delta_{f}=0$. From Eqs. (11) and (13) $)$ one can see that a "flavorless" mixture must be equivalent to either of the following:

1. A mixture of (any) two orthogonal states with equal abundances $(\Delta=0)$, i.e. $\rho=I / 2$. A special case of such mixtures is the usual ansatz for the initial state $(\theta=\Delta=0)$. Note that once $\Delta=0$, the other mixture parameters $(\theta, \alpha)$ become physically insignificant. 
2. Mixtures of the two states: $|\tilde{N}\rangle \pm e^{i \alpha}\left|\tilde{N}^{\dagger}\right\rangle\left(\theta=45^{\circ}\right)$. This is a two parameter family of mixtures, parametrized by $\alpha$ and $\Delta$. In the absence of $\mathrm{CP}$ violation, such a mixture would be a mixture of the $\mathrm{CP}$ eigenstates, analogous to the states $\left|K^{0}\right\rangle \pm\left|\overline{K^{0}}\right\rangle$ in the neutral kaons. Note that for a small CP violation in mixing, which is assumed throughout this work, the difference between these states and the Hamiltonian states is very small, of order $\epsilon \sim \epsilon_{S} / x$.

Note that "flavorless" does not stand for "with vanishing lepton number", as both $\tilde{N}$ and $\tilde{N}^{\dagger}$ decay to both positive and negative lepton number products. In the next section we calculate the lepton asymmetry due to a generic initial mixture, with an emphasis on these "flavorless" mixtures.

\section{LEPTON ASYMMETRIES}

If we define $|\psi(t)\rangle$ and $|\bar{\psi}(t)\rangle$ to be the states which have evolved from $|\psi\rangle$ and $|\bar{\psi}\rangle$ at time $t=0$, respectively, then the time integrated decay rate of $|\psi(t)\rangle$ into a final state $|f\rangle$ is given by

$$
\Gamma_{f}=\mathcal{N} \int_{0}^{\infty}|\langle f|\mathcal{H}| \psi(t)\rangle|^{2} d t,
$$

where $\mathcal{H}$ is the decay Hamiltonian. The lepton asymmetry is given by

$$
\varepsilon \equiv \frac{n\left(\Gamma_{\tilde{L}}+\Gamma_{L}-\Gamma_{\tilde{L}^{\dagger}}-\Gamma_{\bar{L}}\right)+\bar{n}\left(\bar{\Gamma}_{\tilde{L}}+\bar{\Gamma}_{L}-\bar{\Gamma}_{\tilde{L}^{\dagger}}-\bar{\Gamma}_{\bar{L}}\right)}{n\left(\Gamma_{\tilde{L}}+\Gamma_{L}+\Gamma_{\tilde{L}^{\dagger}}+\Gamma_{\bar{L}}\right)+\bar{n}\left(\bar{\Gamma}_{\tilde{L}}+\bar{\Gamma}_{L}+\bar{\Gamma}_{\tilde{L}^{\dagger}}+\bar{\Gamma}_{\bar{L}}\right)},
$$

where $\Gamma_{X}\left(\bar{\Gamma}_{X}\right)$ are the time integrated decay rates of $|\psi(t)\rangle(|\bar{\psi}(t)\rangle)$ into a final state which includes $X$.

We find that an initial mixture of $\psi$ and $\bar{\psi}$ with abundances $n$ and $\bar{n}$ yields a final asymmetry

$$
\varepsilon_{\psi}=\delta_{\tilde{L} \bar{L}}\left[a_{1} \Delta+\left(b_{0}+b_{1} \Delta+b_{2} \Delta^{2}\right) \epsilon\right]+\mathcal{O}\left(\epsilon^{2}\right),
$$

where

$$
\delta_{\tilde{L} \bar{L}} \equiv \frac{\tilde{\mathcal{N}}\left|A_{\tilde{L}}\right|^{2}-\mathcal{N}\left|A_{\bar{L}}\right|^{2}}{\tilde{\mathcal{N}}\left|A_{\tilde{L}}\right|^{2}+\mathcal{N}\left|A_{\bar{L}}\right|^{2}}
$$

is a supersymmetry breaking factor which is of order one at a temperature of $T \sim M(\tilde{\mathcal{N}}$ and $\mathcal{N}$ are phase space factors), and

$$
\begin{aligned}
& a_{1}=-\frac{1-y^{2}}{1+x^{2}}\left[\frac{c-\sin (\alpha-\phi) s x}{1+\cos (\alpha-\phi) s y \Delta}\right], \\
& b_{0}=\frac{x^{2}+y^{2}}{2\left(1+x^{2}\right)}\left[\frac{1}{1+\cos (\alpha-\phi) s y \Delta}\right],
\end{aligned}
$$




$$
\begin{aligned}
b_{1} & =\frac{\cos (\alpha-\phi) s y}{2[1+\cos (\alpha-\phi) s y \Delta]}, \\
b_{2} & =\frac{1}{2}\left(\frac{1-y^{2}}{1+x^{2}}\right)^{2} \frac{c-\sin (\alpha-\phi) s x}{[1+\cos (\alpha-\phi) s y \Delta]^{2}}\left[\sin (\alpha-\phi) s x+c \frac{x^{2}+y^{2}}{1-y^{2}}\right] .
\end{aligned}
$$

The asymmetry due to the usually assumed initial mixture is recovered by substituting $\Delta=\theta=0$. Note that the coefficients $a_{1}, b_{0}, b_{1}$ and $b_{2}$ depend on $\Delta$. An important observation is the absence of the coefficient " $a_{0}$ ", which means that the dynamical contribution $(\Delta=0)$ to the asymmetry is proportional to the parameter of $\mathrm{CP}$ violation in mixing $\epsilon$. This is due to the approximations we have made in the previous section. Since $|\Delta|<1$, the most important coefficients are $a_{1}$ and $b_{0}$. While $b_{0}$ determines the asymmetry for $\Delta=0, a_{1}$ is dominant if $|\Delta| \gg \epsilon$.

We now turn to the special cases of flavorless initial mixtures. First, consider the usual ansatz, namely a mixture of $|\tilde{N}\rangle$ and $\left|\tilde{N}^{\dagger}\right\rangle$ with equal abundances. The resulting asymmetry,

$$
\varepsilon_{\mathrm{eq}}=\frac{1}{2} \delta_{\tilde{L} \bar{L}}\left(\frac{x^{2}+y^{2}}{1+x^{2}}\right) \epsilon,
$$

is given by substituting $\theta=\Delta=0$ in Eqs. (16, 18). This agrees with [8, 9]. Recall that this ansatz corresponds to an equal mixture of $|\tilde{N}\rangle$ and $\left|\tilde{N}^{\dagger}\right\rangle$ or of any other orthogonal pair of states, as explained in the previous section.

As discussed in the previous section, a completely distinct flavorless initial state is given by $\theta=45^{\circ}$. Such a mixture is composed of the approximate CP eigenstates. Note that this basis of states deviates from the Hamiltonian eigenbasis by $\mathcal{O}(\epsilon)$. If the high energy theory is CP conserving, such a mixture is expected to be produced. For this special case, Eq. (18) yields

$$
\begin{aligned}
a_{1} & =\frac{1-y^{2}}{1+x^{2}}\left[\frac{\sin (\alpha-\phi) x}{1+\cos (\alpha-\phi) y \Delta}\right], \\
b_{0} & =\frac{x^{2}+y^{2}}{2\left(1+x^{2}\right)}\left[\frac{1}{1+\cos (\alpha-\phi) y \Delta}\right], \\
b_{1} & =\frac{\cos (\alpha-\phi) y}{2[1+\cos (\alpha-\phi) y \Delta]}, \\
b_{2} & =-\frac{1}{2}\left(\frac{1-y^{2}}{1+x^{2}}\right)^{2}\left[\frac{\sin (\alpha-\phi) x}{1+\cos (\alpha-\phi) y \Delta}\right]^{2} .
\end{aligned}
$$

Unlike the former case, here $a_{1} \neq 0$, and thus the lepton asymmetry is enhanced by $\mathcal{O}(1 / \epsilon)$ compared to the former case. Note that $a_{1}$ does vanish for $\alpha=\phi$.

Concerning an unbalanced mixture of the flavor states, there is an interesting obser- 
vation: Substituting $\theta=0$ in Eq. (18) we obtain

$$
\begin{aligned}
a_{1} & =-\frac{1-y^{2}}{1+x^{2}}, \\
b_{0} & =\frac{x^{2}+y^{2}}{2\left(1+x^{2}\right)}, \\
b_{1} & =0 \\
b_{2} & =\frac{\left(1-y^{2}\right)\left(x^{2}+y^{2}\right)}{2\left(1+x^{2}\right)^{2}} .
\end{aligned}
$$

Note that $b_{1}$ vanishes and that both $a_{1}$ and $b_{2}$ are proportional to $1-y^{2}$. Therefore, at least to order $\epsilon$, all the contribution due to the initial asymmetry ( $\Delta$ terms) is completely washed out once one of the mass eigenstates is very long lived. Actually, taking the $y^{2} \rightarrow 1$ limit recovers the asymmetry (9) of the usually assumed initial state. Namely, to order $\epsilon$, if one of the states is very long lived compared with the other, the produced asymmetry does not depend on the initial asymmetry. This effect is not significant for Soft Leptogenesis if we assume that $y=\mathcal{O}\left(\epsilon_{S}\right) \ll 1$ (see [11]). It would be significant in models where $y \approx 1-\mathcal{O}(\epsilon)$. It might be relevant in other mixing driven mechanisms of asymmetry production.

So far we have discussed only mixtures of two orthogonal states. However, as argued in the previous section, every conceivable mixture (e.g. a mixture of two non orthogonal states, or a mixture of three states, etc.) is always equivalent to a mixture of two orthogonal states such as the one given by Eq. (10). Thus, given a generic mixture, we would like to find the equivalent orthogonal mixture. This can be done by diagonalizing the density matrix. The two orthogonal states $|\psi\rangle$ and $|\bar{\psi}\rangle$ are simply the eigenvectors, and the abundances $n$ and $\bar{n}$ are given by the corresponding eigenvalues.

A particularly simple example for the above is a mixture of the Hamiltonian eigenstates $\left|\tilde{N}_{L}\right\rangle$ and $\left|\tilde{N}_{H}\right\rangle$, where $L, H$ stand for light,heavy respectively. The lepton asymmetry due to such a mixture is

$$
\varepsilon_{M}=\frac{1}{4} \delta_{\tilde{L} \bar{L}}(2 \Delta+\epsilon)
$$

where $\Delta$ is the difference between the abundances of $\left|\tilde{N}_{L}\right\rangle$ and $\left|\tilde{N}_{H}\right\rangle$. This result is most easily obtained by directly calculating Eq. (15), rather than by diagonalizing the density matrix, as described above. In any case, this mixture has $\Delta_{f} \neq 0$, owing to the fact that when $|q / p| \neq 0$, both $\left|\tilde{N}_{L}\right\rangle$ and $\left|\tilde{N}_{H}\right\rangle$ have the same asymmetric imbalance between $|\tilde{N}\rangle$ and $\left|\tilde{N}^{\dagger}\right\rangle$. This is analogous to $\mathrm{CP}$ violation in meson mixing, where the Hamiltonian eigenstates (e.g. $K_{S, L}$ ) have the same imbalance as the one between the weak eigenstates (e.g. $K^{0}, \bar{K}^{0}$ accordingly). Note that if the plasma evolves according to the same Hamiltonian, then indeed one would expect decoherence effects to project the 
system on the Hamiltonian basis.

\section{SUMMARY}

We have obtained the final lepton asymmetry due to the most general initial state in the Soft Leptogenesis scenario [8, 10, 11]. We have found that a finite lepton asymmetry is generally produced in Soft Leptogenesis, which we interpret as robustness of the Soft Leptogenesis idea against variations in the high energy theory. However, since the actual amount of this asymmetry depends strongly on the initial state, it seems that the Soft Leptogenesis framework loses some of its predictive power.

Defining $\Delta_{f} \equiv\left\langle n_{f}\right\rangle-\left\langle\bar{n}_{f}\right\rangle$ to be the difference between the expectation values of the sneutrino and anti-sneutrino initial abundances, Eqs. (16) and (A4) demonstrate that the most general initial state yields both a $\Delta_{f}$-independent contribution and an $\epsilon_{S}$-independent one, where $\epsilon_{S} \equiv m_{\mathrm{SUSY}} / M_{N}$. Since these two terms are not related, we may say that a non vanishing final asymmetry is a generic feature of Soft Leptogenesis, regardless of the type of $\mathrm{CP}$ violation involved.

Initial states which are "flavorless" $\left(\Delta_{f}=0\right)$ must either have $\rho=I / 2$ (as in the commonly assumed initial state), or be equivalent to any mixture of the "CP states" $\left(\theta=45^{\circ}\right)$. While in the former case the asymmetry is suppressed by $\epsilon_{S}$, in the latter case it is proportional only to $\Delta$, the difference between the abundances in the mixture.

Another observation is that for an unbalanced initial state $\left(\Delta_{f} \neq 0\right)$, CP violation in mixing between the heavy sneutrinos washes out part of the initial asymmetry in the sneutrino system. If the mixture is equivalent to a mixture of the flavor states, then Eq. (13) shows that this washout gets strong when one of the mass eigenstates becomes very long lived compared to the other $(\Delta \Gamma \approx 2 \Gamma)$. In this limit the system behaves in a way which is similar to the usually assumed case.

As a final remark, we note that the initial state may be of relevance also with regard to other issues, such as: Resonant Leptogenesis [6], flavor [15] and washout mechanisms [16], thermal considerations [17], and scattering effects [18]. Moreover, as noted in [19], there

are effects from quantum Boltzmann equations such as memory effects due to interference, which are enhanced in resonance and mixing induced Leptogenesis. Further research should be done in order to fully understand the relation between these effects and the initial conditions. 


\section{APPENDIX A: OTHER SOURCES OF CP VIOLATION}

In the text above we have considered $\tilde{N}-\tilde{N}^{\dagger}$ mixing as the only source of CP violation. However, as shown in [11], it may not be the only effect once gaugino masses and interactions are taken into account. New contributions arise, which are related to CP violation in decay and in the interference between decays in the presence of $\tilde{N}-\tilde{N}^{\dagger}$ oscillations. These contributions may even be the leading effect for some regions in the parameter space [11]. Considering a general initial state, we expect also these contributions to have an unsuppressed piece proportional to $\Delta$. We verify this behavior of the lepton asymmetry for "flavorless" initial mixtures by calculating the new contributions. We expect similar results to hold in the general case. The calculation is carried out along the same lines as in the previous section, namely: obtaining the new integrated decay rates and substituting them in Eq. (15).

We start with some definitions. Following [11], we add to the Lagrangian a mass term for the Wino,

$$
\mathcal{L}=m_{2} \tilde{W}^{a} \tilde{W}^{a}+\text { c.c. }
$$

where $a=1,2,3$ is a weak isospin index. As in [11], we ignore the Bino since it would have similar effects. Note that since $m_{2}$ is generally complex, there is one more weak phase in the Lagrangian, $\phi_{W} \equiv \arg \left(m_{2} M B^{*}\right)$. Other useful definitions are

$$
\begin{gathered}
\sin \delta_{s} \equiv \sin \frac{\arg \left(\lambda_{\tilde{L}} \lambda_{L^{\dagger}}^{-1}\right)}{2}=\mathcal{O}\left(\alpha_{2}\right), \\
\sin \delta_{f} \equiv \sin \frac{\arg \left(\lambda_{\bar{L}} \lambda_{L}^{-1}\right)}{2}=\mathcal{O}(1) .
\end{gathered}
$$

We also relax the approximation $A_{\bar{L}}=\bar{A}_{L}$, and consider non vanishing values for $\bar{A}_{\tilde{L}}, A_{\tilde{L}^{\dagger}}$ and $A_{L}, \bar{A}_{\bar{L}}$. We also note that according to the assumptions of [11], it follows that

$$
\epsilon \sim \epsilon_{S} / x
$$

where $\epsilon_{S} \equiv m_{\mathrm{SUSY}} / M$. More details can be found in [11].

Next we calculate the lepton asymmetries for the flavorless "CP" mixtures $\left(\theta=45^{\circ}\right)$. In what follows, we ignore $\mathcal{O}(1)$ factors which are similar to $\delta_{\tilde{L} \bar{L}}$ defined in Eq.(17). Calculating the integrated decay rates and substituting in Eq. (15), we obtain a lepton asymmetry of the form

$$
\varepsilon=a_{1} \Delta+\left(b_{0}+b_{1} \Delta+\ldots\right) \epsilon_{S}+\left(c_{0}+c_{1} \Delta+\ldots\right) \epsilon_{S}^{2}+\mathcal{O}\left(\epsilon_{S}^{3}\right)
$$

where: 
1. The term $a_{1} \Delta$ is the expected piece due to generalizing the initial state. We find that

$$
a_{1}=-\frac{x \sin (\alpha-\phi)}{2\left(1+x^{2}\right)} .
$$

2. The term $b_{0} \epsilon_{S}$ is given by Eq.(19), confirming the result of [8]. This contribution is due to $\mathrm{CP}$ violation in mixing.

3. The term $c_{0} \epsilon_{S}^{2}$ can be divided into various contributions according to the type of $\mathrm{CP}$ violation involved:

- Another contribution from $\mathrm{CP}$ violation in mixing:

$$
\begin{aligned}
\varepsilon_{2}^{m} & =\frac{\epsilon x}{2\left(1+x^{2}\right)}\left[\left(\left|\frac{\bar{A}_{\tilde{L}}}{A_{\tilde{L}}}\right|+\left|\frac{A_{\tilde{L}^{\dagger}}}{\bar{A}_{\tilde{L}^{\dagger}}}\right|\right) \sin \delta_{s} \cos \phi-\left(\left|\frac{A_{L}}{\bar{A}_{L}}\right|+\left|\frac{\bar{A}_{\bar{L}}}{A_{\bar{L}}}\right|\right) \sin \delta_{f} \cos \phi_{W}\right] \\
& =\mathcal{O}\left(\frac{\alpha_{2} x \epsilon_{S}^{2}}{1+x^{2}}\right)
\end{aligned}
$$

- $\mathrm{CP}$ violation in decay:

$$
\varepsilon^{d}=\frac{y}{2}\left(\left|\frac{\bar{A}_{\tilde{L}}}{A_{\tilde{L}}}\right|-\left|\frac{A_{\tilde{L}^{\dagger}}}{\bar{A}_{\tilde{L}^{\dagger}}}\right|\right) \cos \delta_{s} \cos \phi=\mathcal{O}\left(\alpha_{2} \epsilon_{S}^{2}\right) .
$$

Note that this contribution vanishes in the absence of mixing, since it is proportional to $y$. The fact that there is no contribution due to pure $\mathrm{CP}$ violation in decay is because such a pure direct $\mathrm{CP}$ violation term,

$$
\varepsilon_{2}^{d}=\frac{\mathcal{N}_{s}\left(\left|A_{\tilde{L}^{\dagger}}\right|^{2}-\left|\bar{A}_{\tilde{L}}\right|^{2}\right)+\mathcal{N}_{f}\left(\left|\bar{A}_{L}\right|^{2}-\left|A_{\bar{L}}\right|^{2}\right)}{\mathcal{N}_{s}\left(\left|A_{\tilde{L}}\right|^{2}+\left|\bar{A}_{\tilde{L}^{\dagger}}\right|^{2}\right)+\mathcal{N}_{f}\left(\left|\bar{A}_{L}\right|^{2}+\left|A_{\bar{L}}\right|^{2}\right)}
$$

vanishes to $\mathcal{O}\left(\epsilon_{S}^{2}\right)$ due to the equality

$$
\left|A_{\tilde{L}}\right|=\left|\bar{A}_{\tilde{L}^{\dagger}}\right|, \quad\left|A_{L}\right|=\left|\bar{A}_{\bar{L}}\right|
$$

which holds to $\mathcal{O}\left(\epsilon_{S}^{2}\right)$, and due to the CPT theorem, which asserts that

$$
\mathcal{N}_{s}\left(\left|A_{\tilde{L}}\right|^{2}+\left|A_{\tilde{L}^{\dagger}}\right|^{2}\right)+\mathcal{N}_{f}\left(\left|A_{L}\right|^{2}+\left|A_{\bar{L}}\right|^{2}\right)=\mathcal{N}_{s}\left(\left|\bar{A}_{\tilde{L}}\right|^{2}+\left|\bar{A}_{\tilde{L}^{\dagger}}\right|^{2}\right)+\mathcal{N}_{f}\left(\left|\bar{A}_{L}\right|^{2}+\left|\bar{A}_{\bar{L}}\right|^{2}\right) .
$$

- $\mathrm{CP}$ violation in the interference between two decays differing by mixing:

$$
\begin{aligned}
\varepsilon^{i} & =\frac{y}{2}\left[\left(\left|\frac{\bar{A}_{\tilde{L}}}{A_{\tilde{L}}}\right|+\left|\frac{A_{\tilde{L}^{\dagger}}}{\bar{A}_{\tilde{L}^{\dagger}}}\right|\right) \sin \delta_{s} \sin \phi-\left(\left|\frac{\bar{A}_{\bar{L}}}{A_{\bar{L}}}\right|+\left|\frac{A_{L}}{\bar{A}_{L}}\right|\right) \sin \delta_{f} \sin \phi_{W}\right] \\
& =\mathcal{O}\left(\alpha_{2} \epsilon_{S}^{2}\right) .
\end{aligned}
$$


- CP violation from combined sources:

$$
\varepsilon^{m d i}=-\frac{\epsilon x}{2\left(1+x^{2}\right)}\left(\left|\frac{\bar{A}_{\tilde{L}}}{A_{\tilde{L}}}\right|-\left|\frac{A_{\tilde{L}^{\dagger}}}{\bar{A}_{\tilde{L}^{\dagger}}}\right|\right) \cos \delta_{s} \sin \phi=\mathcal{O}\left(\frac{\alpha_{2} x \epsilon_{S}^{2}}{1+x^{2}}\right) .
$$

This confirms the results of [11], up to a minus sign in Eqs. (A7A11).

4. The coefficients $b_{1}, c_{1}, \ldots$ signify contributions proportional to both $\Delta$ and $\epsilon_{S}$. Since $\epsilon_{S} \ll 1$, their effect is negligible compared to that of $a_{1}$.

This demonstrates that Soft Leptogenesis generally predicts a lepton asymmetry for any initial state, irrespective of the type of $\mathrm{CP}$ violation involved.

\section{ACKNOWLEDGMENTS}

We thank Mu-Chun Chen for asking the question which led to the investigation of initial state effects. We also thank Yuval Grossman and Yossi Nir for useful discussions and for reading the manuscript.

[1] A. D. Sakharov, Pisma Zh. Eksp. Teor. Fiz. 5, 32 (1967) [JETP Lett. 5, 24 (1967 SOPUA,34,392-393.1991 UFNAA,161,61-64.1991)].

[2] M. Fukugita and T. Yanagida, Phys. Lett. B 174, 45 (1986).

[3] W. Buchmuller and M. Plumacher, Phys. Lett. B 431, 354 (1998) arXiv:hep-ph/9710460; A. Pilaftsis, Int. J. Mod. Phys. A 14, 1811 (1999) arXiv:hep-ph/9812256]; W. Buchmuller, P. Di Bari and M. Plumacher, New J. Phys. 6, 105 (2004) arXiv:hep-ph/0406014.

[4] B. A. Campbell, S. Davidson and K. A. Olive, Nucl. Phys. B 399, 111 (1993) arXiv:hep-ph/9302223.

[5] L. Covi, E. Roulet and F. Vissani, Phys. Lett. B 384, 169 (1996) arXiv:hep-ph/9605319; R. Allahverdi, B. Dutta and A. Mazumdar, Phys. Rev. D 67, 123515 (2003) arXiv:hep-ph/0301184.

[6] M. Flanz, E. A. Paschos and U. Sarkar, Phys. Lett. B 345, 248 (1995) [Erratum-ibid. B 382, 447 (1996)] arXiv:hep-ph/9411366]; M. Flanz, E. A. Paschos, U. Sarkar and J. Weiss, Phys. Lett. B 389, 693 (1996) arXiv:hep-ph/9607310; A. Pilaftsis, Nucl. Phys. B 504, 61 (1997) arXiv:hep-ph/9702393, Phys. Rev. D 56, 5431 (1997) arXiv:hep-ph/9707235; A. Pilaftsis and T. E. J. Underwood, Nucl. Phys. B 692, 303 (2004) arXiv:hep-ph/0309342, Phys. Rev. D 72, 113001 (2005) arXiv:hep-ph/0506107]; T. Hambye, J. March-Russell and S. M. West, JHEP 0407, 070 (2004) arXiv:hep-ph/0403183. 
[7] L. Covi and E. Roulet, Phys. Lett. B 399, 113 (1997) arXiv:hep-ph/9611425.

[8] Y. Grossman, T. Kashti, Y. Nir and E. Roulet, Phys. Rev. Lett. 91, 251801 (2003) arXiv:hep-ph/0307081.

[9] G. D'Ambrosio, G. F. Giudice and M. Raidal, Phys. Lett. B 575, 75 (2003) arXiv:hep-ph/0308031.

[10] L. Boubekeur, T. Hambye and G. Senjanovic, Phys. Rev. Lett. 93, 111601 (2004) arXiv:hep-ph/0404038; G. D'Ambrosio, T. Hambye, A. Hektor, M. Raidal and A. Rossi, Phys. Lett. B 604, 199 (2004) arXiv:hep-ph/0407312]; E. J. Chun and S. Scopel, Phys. Lett. B 636, 278 (2006) arXiv:hep-ph/0510170; A. D. Medina and C. E. M. Wagner, JHEP 0612, 037 (2006) arXiv:hep-ph/0609052 ; J. Garayoa, M. C. Gonzalez-Garcia and N. Rius, JHEP 0702, 021 (2007) arXiv:hep-ph/0611311; J. R. Ellis and S. K. Kang, arXiv:hep-ph/0505162.

[11] Y. Grossman, T. Kashti, Y. Nir and E. Roulet, JHEP 0411, 080 (2004) arXiv:hep-ph/0407063.

[12] L. Boubekeur, arXiv:hep-ph/0208003.

[13] K. Kumekawa, T. Moroi and T. Yanagida, Prog. Theor. Phys. 92, 437 (1994) arXiv:hep-ph/9405337; T. Asaka, K. Hamaguchi, M. Kawasaki and T. Yanagida, Phys. Lett. B 464, 12 (1999) arXiv:hep-ph/9906366; G. F. Giudice, M. Peloso, A. Riotto and I. Tkachev, JHEP 9908, 014 (1999) arXiv:hep-ph/9905242]; G. Lazarides, Springer Tracts Mod. Phys. 163, 227 (2000) arXiv:hep-ph/9904428]; G. F. Giudice, A. Riotto and A. Zaffaroni, Nucl. Phys. B 710, 511 (2005) arXiv:hep-ph/0408155.

[14] R. Rangarajan and H. Mishra, Phys. Rev. D 61, 043509 (2000) arXiv:hep-ph/9908417].

[15] F. X. Josse-Michaux and A. Abada, JCAP 0710, 009 (2007) arXiv:hep-ph/0703084; A. Abada, S. Davidson, F. X. Josse-Michaux, M. Losada and A. Riotto, JCAP 0604, 004 (2006) arXiv:hep-ph/0601083]; E. Nardi, Y. Nir, E. Roulet and J. Racker, JHEP 0601, 164 (2006) arXiv:hep-ph/0601084; A. Abada, S. Davidson, A. Ibarra, F. X. Josse-Michaux, M. Losada and A. Riotto, JHEP 0609, 010 (2006) arXiv:hep-ph/0605281.

[16] O. Vives, Phys. Rev. D 73, 073006 (2006) arXiv:hep-ph/0512160; G. Engelhard, Y. Grossman, E. Nardi and Y. Nir, Phys. Rev. Lett. 99, 081802 (2007) arXiv:hep-ph/0612187; T. Shindou and T. Yamashita, JHEP 0709, 043 (2007) arXiv:hep-ph/0703183.

[17] G. F. Giudice, A. Notari, M. Raidal, A. Riotto and A. Strumia, Nucl. Phys. B 685, 89 (2004) arXiv:hep-ph/0310123; W. Buchmuller, P. Di Bari and M. Plumacher, Annals Phys. 315, 305 (2005) arXiv:hep-ph/0401240.

[18] E. Nardi, J. Racker and E. Roulet, JHEP 0709, 090 (2007) arXiv:0707.0378 [hep-ph]].

[19] A. De Simone and A. Riotto, JCAP 0708, 002 (2007) arXiv:hep-ph/0703175, JCAP 0708, 013 (2007) [arXiv:0705.2183 [hep-ph]]. 